\title{
Homogeneous Dirichlet condition of an anisotropic degenerate parabolic equation
}

Huashui Zhan*

${ }^{\text {*Correspondence: }}$

huashuizhan@163.com;

2012111007@xmut.edu.cn

School of Applied Mathematics,

Xiamen University of Technology,

Xiamen, 361024, P.R. China

\begin{abstract}
Consider the following anisotropic degenerate parabolic equation:$$
\frac{\partial u}{\partial t}=\frac{\partial}{\partial x_{i}}\left(a^{i j}(u) \frac{\partial u}{\partial x_{j}}\right)+\frac{\partial b_{i}(u)}{\partial x_{i}}, \quad(x, t) \in \Omega \times(0, T),
$$

with the homogeneous Dirichlet boundary value. If the equation is not only degenerate in the interior of $\Omega$, but also on the boundary $\partial \Omega$, the paper discusses how to quote the suitable partly boundary condition to assure the well-posedness of an entropy solution of the equation. In particular, it is possible that the solution of the equation is free from the limitation of the boundary condition.
\end{abstract}

MSC: $35 \mathrm{LL} 65 ; 35 \mathrm{~L} 85 ; 35 R 35$

Keywords: anisotropic degenerate parabolic equation; boundary condition; entropy solution

\section{Introduction}

The paper is to consider the anisotropic degenerate parabolic equation of the form

$$
\frac{\partial u}{\partial t}=\frac{\partial}{\partial x_{i}}\left(a^{i j}(u) \frac{\partial u}{\partial x_{j}}\right)+\frac{\partial b_{i}(u)}{\partial x_{i}}, \quad \text { in } Q_{T}=\Omega \times(0, T)
$$

where $\Omega \subset \mathbb{R}^{N}$ is an open bounded domain and the boundary $\partial \Omega=\Sigma$ is $C^{2},\left(a^{i j}\right)$ is a symmetric matrix with nonnegative characteristic values, i.e., for any $\xi \in \mathbb{R}^{N}$,

$$
a^{i j}=a^{j i}, \quad a^{i j} \xi_{i} \xi_{j} \geq 0,
$$

the pairs of the indices $i, j$ imply the sum from 1 to $N$. Moreover, we assume that

$$
a^{i j}(0)=0 \text {. }
$$

Equation (1.1) arises in many applications, e.g., heat flow in materials with temperature dependent on conductivity, flow in a porous medium,

$$
\frac{\partial u}{\partial t}=\Delta u^{m}
$$


It also arises in the boundary layer theory,

$$
w^{2} w_{\eta \eta}-w_{\tau}-\eta U w_{\xi}+A w_{\eta}+B w=0
$$

where $A, B$ are two known functions derived from the Prandtl system, one can refer to [1] for details. Here and in what follows, we say that equation (1.1) is strongly degenerate if there are interior points in the set $\left\{s \in \mathbb{R}:\left(a^{i j}(s)\right)\right.$ is a degenerate matrix $\}$. Clearly, equation (1.1) is of hyperbolic-parabolic mixed type and might have a discontinuous solution. The posedness of the Cauchy problem of equation (1.1) has been deeply investigated (see [2-14] etc.). At the same time, Li and Wang [15] studied the well-posedness for anisotropic degenerate parabolic equation (1.1) with inhomogeneous boundary condition on a bounded rectangle by using the kinetic formulation which was introduced in [16]. Kobayasi and Ohwa [17] considered the entropy solutions of equation (1.1) with the homogeneous Dirichlet boundary value in an arbitrary bounded domain. Since the entropy solutions defined in $[15,17]$ are only in the $L^{\infty}$ space, the existence of the trace (defined in a traditional way, which was called the strong trace in [17]) on the boundary is not guaranteed, the appropriate definition of entropy solutions is quoted, and a new definition of the trace of the solution on the boundary, defined in an integral formula sense, is introduced; they called it the weak trace. So, not only is Definition 1.1 in what follows different from the definitions of entropy solutions in $[15,17]$, but the trace of the solution in our paper is also in the traditional way.

In fact, if we want to consider the initial boundary value problem of equation (1.1), the initial value condition is always required

$$
u(x, 0)=u_{0}(x), \quad x \in \Omega .
$$

But can we give Dirichlet homogeneous boundary condition

$$
u(x, t)=0, \quad(x, t) \in \partial \Omega \times(0, T)=\Sigma \times(0, T)
$$

as usual?

Clearly, if (1.2) and (1.6) are both true, equation (1.1) is not only degenerate in the interior of $\Omega$, but also degenerate on the boundary $\Sigma$ of $\Omega$. If equation (1.1) is weakly degenerate, we can give the boundary value (1.6) as usual. But if equation (1.1) is strongly degenerate, we shall show that only a portion of the boundary should be given the boundary value. Let us give a basic review of the history of the corresponding problem and show what we consider now.

The memoir by Tricomi [18], as well as subsequent investigations of equations of mixed type, elicited interest in the general study of elliptic equations degenerating on the boundary of the domain. The paper by Keldyš [19] played a significant role in the development of the theory. It was this paper that first brought to light the fact that in the case of elliptic equations degenerating on the boundary, under definite assumptions, a portion of the boundary may be free from the prescription of boundary conditions. Later, Fichera [20, 21] and Oleünik $[22,23]$ developed and perfected the general theory of second order equation with a nonnegative characteristic form, which in particular contains those degenerating on the boundary. 
The equation considered by Fichera and Oleinnik is linear and the second order derivatives of coefficients of principal part are bounded. From Fichera-Oleinnik theory, for a linear degenerate elliptic equation,

$$
a^{r s}(x) \frac{\partial^{2} u}{\partial x_{r} \partial x_{s}}+b_{r}(x) \frac{\partial u}{\partial x_{r}}+c(x) u=f(x), \quad x \in \widetilde{\Omega} \subset \mathbb{R}^{N+1},
$$

where the pairs of the indices $r, s$ imply the sum from 1 to $N+1$. If one wants to consider the boundary value problem of (1.7), one needs and only needs to give a partly boundary condition. In detail, let $\left\{n_{s}\right\}$ be the unit inner normal vector of $\partial \widetilde{\Omega}$ and denote

$$
\begin{aligned}
& \Sigma_{2}=\left\{x \in \partial \widetilde{\Omega}: a^{r s} n_{r} n_{s}=0,\left(b_{r}-a_{x_{s}}^{r s}\right) n_{r}<0\right\}, \\
& \Sigma_{3}=\left\{x \in \partial \widetilde{\Omega}: a^{r s} n_{s} n_{r}>0\right\} .
\end{aligned}
$$

Then, to ensure the posedness of equation (1.7), Fichera-Oleinik theory tells us that the suitable boundary condition is

$$
\left.u\right|_{\Sigma_{2} \cup \Sigma_{3}}=g(x) .
$$

In particular, if the matrix $\left(a^{r s}\right)$ is positive definite, $(1.8)$ is just the usual Dirichlet boundary condition.

Now, for equation (1.3), or the general equation

$$
u_{t}=\triangle A(u)
$$

with the existence of $A^{-1}$, in other words, equation (1.9) is weakly degenerate, then let $v=A(u), u=A^{-1}(v)$,

$$
\Delta v-\left(A^{-1}(v)\right)_{t}=0
$$

According to Fichera-Oleinnik theory, we know that we can give the Dirichlet homogeneous boundary condition (1.6). For equation (1.4), if the domain $\Omega=\{0<\tau<T, 0<\xi<$ $X, 0<\eta<1\}$, then comparing (1.4) with (1.7), according to Fichera-Oleinik theory, the initial and the boundary value conditions for $w$ have the form

$$
\left.w\right|_{\tau=0}=w_{0}(\xi, \eta),\left.\quad w\right|_{\eta=1}=0,\left.\quad\left(v w w_{\eta}-v_{0} w+c(\tau, \xi)\right)\right|_{\eta=0}=0,
$$

where $v$ is the viscous coefficient, $v_{0}$ and $c(\tau, \xi)$ are known functions; one can refer to [1] for details.

But if equation (1.1) is strongly degenerate, then the inverse matrix $A^{-1}=\left(a_{i j}\right)^{-1}$ is not existential, we cannot deal with it as (1.10). Rewrite equation (1.1) as

$$
\frac{\partial u}{\partial t}=a^{i j}(u) \frac{\partial^{2} u}{\partial x_{i} \partial x_{j}}+a^{i j^{\prime}}(u) \frac{\partial u}{\partial x_{i}} \frac{\partial u}{\partial x_{j}}+b_{i}^{\prime}(u) \frac{\partial u}{\partial x_{i}}, \quad \text { in } Q_{T}=\Omega \times(0, T)
$$


the domain is a cylinder $\Omega \times(0, T)$. If we let $t=x_{N+1}$ and regard the degenerate parabolic equation (1.12) as the form of a 'linear' degenerate elliptic equation as in (1.7), then

$$
\left(\widetilde{a}^{r s}\right)_{(N+1) \times(N+1)}=\left(\begin{array}{cc}
a^{i j} & 0 \\
0 & 0
\end{array}\right) .
$$

If $a^{i j}(0)=0$, which means that equation (1.12) is not only strongly degenerate in the interior of $\Omega$, but also degenerate on the boundary $\partial \Omega$, then $\Sigma_{3}$ is an empty set, while

$$
\widetilde{b}_{s}(x, t)= \begin{cases}b_{i}^{\prime}(u)+a^{i j^{\prime}}(u) \frac{\partial u}{\partial x_{j}}, & 1 \leq s=i \leq N, \\ -1, & s=N+1 .\end{cases}
$$

Under this observation, according to Fichera-Oleinnik theory, the initial value condition (1.5) is always needed, but on the lateral boundary $\partial \Omega \times(0, T)$, by $a^{i j}(0)=0$, the partly boundary on which we should give the boundary value is

$$
\begin{aligned}
\Sigma_{p} & =\left\{x \in \partial \Omega:\left(b_{i}^{\prime}(0)+\left.a^{i j^{\prime}}(0) \frac{\partial u}{\partial x_{j}}\right|_{x \in \partial \Omega}-\left.a^{i j^{\prime}}(0) \frac{\partial u}{\partial x_{j}}\right|_{x \in \partial \Omega}\right) n_{i}<0\right\} \\
& =\left\{x \in \partial \Omega: b_{i}^{\prime}(0) n_{i}<0\right\},
\end{aligned}
$$

where $\left\{n_{i}\right\}$ is the unit inner normal vector of $\partial \Omega$.

Though (1.13) seems reasonable and beautiful, whether the term $\left.\frac{\partial u}{\partial x_{i}}\right|_{x \in \partial \Omega}$ has an explicit definition is unclear, unless equation (1.12) has a classical solution. In fact, due to the strongly degenerate property of $\left(a^{i j}\right)$, equation (1.12) generally only has a weak solution. In our paper, we consider the solution of equation (1.12) in $B V$ sense, and we cannot define the trace of $\frac{\partial u}{\partial x_{i}}$ on $\partial \Omega$, which means that we also cannot define

$$
\Sigma_{p}=\left\{x \in \partial \Omega:\left(b_{i}^{\prime}(0)+\left.a^{i j^{\prime}}(0) \frac{\partial u}{\partial x_{j}}\right|_{x \in \partial \Omega}-\left.a^{i j^{\prime}}(0) \frac{\partial u}{\partial x_{j}}\right|_{x \in \partial \Omega}\right) n_{i}<0\right\} .
$$

Fortunately, only if $b_{i}(s)$ is derivable, then

$$
\Sigma_{p}=\left\{x \in \partial \Omega: b_{i}^{\prime}(0) n_{i}<0\right\}
$$

has a definite sense. Our paper will show that $\Sigma_{p}$ defined in (1.14) can be given the boundary condition in some way.

It is well known that the BV functions are the weakest functions which have the traces in the usual way. At the same time, in order to get the uniqueness, we need to consider the entropy solution of equation (1.1) instead of the general weak solution.

The existence will be proved by means of the method of parabolic regularization, namely the solution of our problem will be obtained as a limit point of the family $\left\{u_{\varepsilon}\right\}$ of solutions of the regularized problem

$$
\frac{\partial u}{\partial t}=\frac{\partial}{\partial x_{i}}\left(a^{i j}(u) \frac{\partial u}{\partial x_{j}}\right)+\varepsilon \Delta u+\frac{\partial b_{i}(u)}{\partial x_{i}}, \quad \text { in } Q_{T}=\Omega \times(0, T),
$$

with compatible initial boundary values (1.4)-(1.5). 
In order to prove the compactness of $\left\{u_{\varepsilon}\right\}$, we need some estimates on $\left\{u_{\varepsilon}\right\}$. However, in the present case of strong degeneration, it is difficult to estimate $\left|\operatorname{grad} u_{\varepsilon}\right|_{L^{1}\left(Q_{T}\right)}$. In addition, since for the limit function $u$ of certain subsequence of $\left\{u_{\varepsilon}\right\}, \widehat{a^{i j}(u)} \frac{\partial u}{\partial x_{j}}$ need not have the trace $\gamma\left(\widehat{a^{i j}(u)} \frac{\partial u}{\partial x_{j}}\right)$ on $\Sigma$, we have to make a detour to avoid $\gamma\left(\widehat{a^{i j}(u)} \frac{\partial u}{\partial x_{j}}\right)$ in defining solution, where $\widehat{a^{i j}(u)}$ is the composite means function of BV function $a^{i j}(u)$, which will be defined in detail in what follows. By combining this inspiring idea of [24] with that of [9], we shall give a new entropy solution of equation (1.1). Let us give some preparedness.

For any $\eta>0, \forall k \in R$, let $\vec{n}=\left\{n_{i}\right\}$ be the inner unit normal vector of $\Sigma$, and

$$
\begin{aligned}
& \Sigma_{1 \eta k}=\left\{x \in \Sigma, S_{\eta}(k)\left[b_{i}(0)-b_{i}(k)\right] n_{i}>0\right\}, \\
& \Sigma_{2 \eta k}=\left\{x \in \Sigma, S_{\eta}(k)\left[b_{i}(0)-b_{i}(k)\right] n_{i} \leq 0\right\} .
\end{aligned}
$$

Clearly, $\Sigma=\Sigma_{1 \eta k} \cup \Sigma_{2 \eta k}$, and let

$$
\Sigma_{1}=\bigcup_{\forall \eta>0, \forall k \in \mathbb{R}} \Sigma_{1 \eta k}, \quad \Sigma_{2}=\Sigma \backslash \Sigma_{1}
$$

Now, if $\Sigma_{1} \neq \emptyset$, we can give the boundary value condition as

$$
\left.\gamma u\right|_{\Sigma_{1}}=0
$$

In fact, by the definition of $\Sigma_{1 \eta k}$, we know that

$$
0<S_{\eta}(k)\left[b_{i}(0)-b_{i}(k)\right] n_{i}(x, t)=-k S_{\eta}(k) b_{i}^{\prime}(\zeta) n_{i}(x, t),
$$

where $\zeta \in(k, 0)$. If we let $\eta \rightarrow 0$, then

$$
b_{i}^{\prime}(\zeta) n_{i}(x, t)<0 .
$$

Let $k \rightarrow 0$. We know that

$$
b_{i}^{\prime}(0) n_{i}(x, t)<0,
$$

which is in accordance with (1.14).

Let us consider $\Sigma_{1} \neq \emptyset$ firstly. As for the case of $\Sigma_{1}=\emptyset$, no boundary value condition is necessary. In other words, the solution of equation (1.1) is completely controlled by the initial value condition. We shall discuss the problem in this case at the end of the paper.

Let $S_{\eta}(s)=\int_{0}^{s} h_{\eta}(\tau) d \tau$ for small $\eta>0$, where $h_{\eta}(s)=\frac{2}{\eta}\left(1-\frac{|s|}{\eta}\right)_{+}$. Obviously, $h_{\eta}(s) \in C(\mathbb{R})$, and

$$
h_{\eta}(s) \geq 0, \quad\left|s h_{\eta}(s)\right| \leq 1, \quad\left|S_{\eta}(s)\right| \leq 1 ; \quad \lim _{\eta \rightarrow 0} S_{\eta}(s)=\operatorname{sgn} s, \quad \lim _{\eta \rightarrow 0} s S_{\eta}^{\prime}(s)=0 .
$$

Definition 1.1 If $\Sigma_{1} \neq \emptyset$, a function $u$ is said to be the entropy solution of equation (1.1)(1.5)-(1.19), if 
1. $u \in B V\left(Q_{T}\right) \cap L^{\infty}\left(Q_{T}\right)$, and there exist the functions $g^{i} \in L^{2}\left(Q_{T}\right), i=1,2, \ldots, N$, such that

$$
\iint_{Q_{T}} g^{i}(x, t) \varphi(x, t) d x d t=\iint_{Q_{T}} \widehat{\gamma}^{i j}(u) \varphi(x, t) \frac{\partial u}{\partial x_{j}} d x d t
$$

where $\varphi(x, t) \in L^{2}\left(Q_{T}\right),\left(\gamma^{i j}\right)$ is the square root of $\left(a^{i j}\right)$, and

$$
\widehat{\gamma}^{i j}(u)=\int_{0}^{1} \gamma^{i j}\left(s u^{+}+(1-s) u^{-}\right) d s .
$$

2. For any $\varphi_{1}, \varphi_{2} \in C^{2}\left(\overline{Q_{T}}\right), \varphi_{1} \geq 0,\left.\nabla \varphi_{1}\right|_{\Sigma}=0,\left.\varphi_{1}\right|_{\partial \Omega \times[0, T]}=\left.\varphi_{2}\right|_{\partial \Omega \times[0, T]}$, and $\operatorname{supp} \varphi_{2}$, $\operatorname{supp} \varphi_{1} \subset \bar{\Omega} \times(0, T)$, for any $k \in \mathbb{R}$, for any small $\eta>0$, $u$ satisfies

$$
\begin{aligned}
& \iint_{Q_{T}}\left[I_{\eta}(u-k) \varphi_{1 t}-B_{\eta}^{i}(u, k) \varphi_{1 x_{i}}+A_{\eta}^{i j}(u, k) \frac{\partial \varphi_{1}}{\partial x_{i}} \frac{\partial \varphi_{1}}{\partial x_{j}}-S_{\eta}^{\prime}(u-k) \sum_{j=1}^{N}\left|g^{j}\right|^{2} \varphi_{1}\right] d x d t \\
& \quad+S_{\eta}(k) \iint_{Q_{T}}\left[u \varphi_{2 t}-\left(b_{i}(u)-b_{i}(0)\right) \varphi_{2 x_{i}}+A^{i j}(u) \frac{\partial \varphi_{2}}{\partial x_{i}} \frac{\partial \varphi_{2}}{\partial x_{j}}\right] d x d t \\
& \quad+S_{\eta}(k) \int_{0}^{T} \int_{\Sigma_{1 \eta k}}\left[\left(b_{i}(0)-b_{i}(k)\right] n_{i} \varphi_{1} d t d \sigma \geq 0\right.
\end{aligned}
$$

3. The boundary value is satisfied in the sense of the trace

$$
\left.\gamma u\right|_{\Sigma_{1 \eta k}}=0
$$

4. The initial value is satisfied in the sense of the following equality:

$$
\lim _{t \rightarrow 0} \int_{\Omega}\left|u(x, t)-u_{0}(x)\right| d x=0, \quad \text { a.e. } x \in \Omega,
$$

where the pairs of equal indices imply a summation from 1 up to $N$, and

$$
\begin{array}{ll}
B_{\eta}^{i}(u, k)=\int_{k}^{u} b_{i}^{\prime}(s) S_{\eta}(s-k) d s, & I_{\eta}(u-k)=\int_{0}^{u-k} S_{\eta}(s) d s, \\
A_{\eta}^{i j}(u, k)=\int_{k}^{u} a^{i j}(s) S_{\eta}(s-k) d s, & A^{i j}(u)=\int_{0}^{u} a^{i j}(s) d s .
\end{array}
$$

Clearly, let $\eta \rightarrow 0$ in (1.21). We can see that if $u$ is the entropy solution in Definition 1.1, then it is an entropy solution defined in $[2,3,7]$ etc.

We shall prove the following theorems.

Theorem 1.2 Suppose that $A^{i j}(s)$ is $C^{3}, b_{i}(s)$ is $C^{2}$, and $u_{0}(x) \in L^{\infty}(\Omega) \cap C^{2}(\Omega)$, and suppose that

$$
a^{i j}(0)=0 \text {. }
$$

Then equation (1.1) with initial boundary value conditions (1.5), (1.19) has an entropy solution in the sense of Definition 1.1. 
Theorem 1.3 Suppose that $A^{i j}(s)$ and $b_{i}(s)$ are $C^{1}$. Let $u, v$ be solutions of equation (1.1) with different initial values $u_{0}(x), v_{0}(x) \in L^{\infty}(\Omega)$, respectively. Suppose that

$$
\gamma u(x, t)=f(x, t), \quad \gamma v=g(x, t), \quad(x, t) \in \Sigma \times(0, T),
$$

and in particular

$$
\gamma u=\gamma v=0, \quad x \in \Sigma_{1}
$$

suppose that the distance function $d(x)=\operatorname{dist}(x, \Sigma)<\lambda$ satisfies

$$
\left|d_{x_{i} x_{j}}\right| \leq c
$$

where $\lambda$ is a small enough constant, and $\Omega_{\lambda}=\{x \in \Omega, d(x, \partial \Omega)<\lambda\}$. Then

$$
\int_{\Omega}|u(x, t)-v(x, t)| d x \leq \int_{\Omega}\left|u_{0}-v_{0}\right| d x+\underset{(x, t) \in \Sigma_{2} \times(0, T)}{\operatorname{ess} \sup _{1}}|f(x, t)-g(x, t)|,
$$

where $(x, t) \in \mathbb{R}^{N+1}$, ess $\sup _{(x, t) \in \Sigma_{2} \times(0, T)}|f(x, t)-g(x, t)|$ is in the sense of $N$-dimensional Hausdorff measure.

\section{The proof of the existence}

Without loss of generality, one may assume that $u \in B V\left(Q_{T}\right)$ is an almost everywhere continuous function on $Q_{T}$.

Let $\Gamma_{u}$ be the set of all jump points of $u \in B V\left(Q_{T}\right)$, let $v=\left(v_{1}, v_{2}, \ldots, v_{N}, v_{N+1}\right)$ be the normal of $\Gamma_{u}$ at $X=(x, t), u^{+}(X)$, and let $u^{-}(X)$ be the approximate limits of $u$ at $X \in \Gamma_{u}$ with respect to $(v, Y-X)>0$ and $(v, Y-X)<0$, respectively. For a continuous function $p(u, x, t)$ and $u \in B V\left(Q_{T}\right)$, define

$$
\widehat{p}(u, x, t)=\int_{0}^{1} p\left(\tau u^{+}+(1-\tau) u^{-}, x, t\right) d \tau,
$$

which is called the composite mean value of $p$. For a given $t$, we denote $\Gamma_{u}^{t}, H^{t},\left(v_{1}^{t}, \ldots, v_{N}^{t}\right)$ and $u_{ \pm}^{t}$ as all jump points of $u(\cdot, t)$, Hausdorff measure of $\Gamma_{u}^{t}$, the unit normal vector of $\Gamma_{u}^{t}$, and the asymptotic limit of $u(\cdot, t)$, respectively. Moreover, if $f(s) \in C^{1}(\mathbb{R}), u \in B V\left(Q_{T}\right)$, then $f(u) \in B V\left(Q_{T}\right)$ and

$$
\frac{\partial f(u)}{\partial x_{i}}=\widehat{f}^{\prime}(u) \frac{\partial u}{\partial x_{i}}, \quad i=1,2, \ldots, N .
$$

Lemma 2.1 [25] Assume that $\Omega \subset \mathbb{R}^{N}$ is an open bounded set, and let $f_{k}, f \in L^{q}(\Omega)$, as $k \rightarrow \infty, f_{k} \rightarrow f$ weakly in $L^{q}(\Omega), 1 \leq q<\infty$. Then

$$
\liminf _{k \rightarrow \infty}\left\|f_{k}\right\|_{L^{q}(\Omega)}^{q} \geq\|f\|_{L^{q}(\Omega)}^{q} .
$$

Lemma 2.2 [24] Let $u_{\varepsilon}$ be a solution of equation (1.15) with initial boundary value (1.5), (1.6). If the assumptions of Theorem 1.2 are true, then 


$$
\varepsilon \int_{\Sigma}\left|\frac{\partial u_{\varepsilon}}{\partial n}\right| d \sigma \leq c_{1}+c_{2}\left(\left|\nabla u_{\varepsilon}\right|_{L^{1}(\Omega)}+\left|\frac{\partial u_{\varepsilon}}{\partial t}\right|_{L^{1}(\Omega)}\right)
$$

with constants $c_{i}, i=1,2$, independent of $\varepsilon$.

Under the assumptions of $A, b_{i}$ and $u_{0}$ in Theorem 1.2, it is well known that there is a classical solution $u_{\varepsilon}$ of the initial boundary value problem (1.15)-(1.5)-(1.6), e.g., one can refer to Chapter 8 of [26].

We need to make some estimates for $u_{\varepsilon}$ of (1.15). Firstly, since $u_{0}(x) \in L^{\infty}(\Omega)$ is suitably smooth, by the maximum principle, we have

$$
\left|u_{\varepsilon}\right| \leq\left\|u_{0}\right\|_{L^{\infty}} \leq M
$$

Secondly, let us make the $B V$ estimates on $u_{\varepsilon}$.

Theorem 2.3 Let $u_{\varepsilon}$ be a solution of (1.15) with initial boundary value conditions (1.5), (1.6). If the assumptions of Theorem 1.2 are true, then

$$
\left|\operatorname{grad} u_{\varepsilon}\right|_{L^{1}(\Omega)} \leq c
$$

where $|\operatorname{grad} u|^{2}=\sum_{i=1}^{N}\left|\frac{\partial u}{\partial x_{i}}\right|^{2}+\left|\frac{\partial u}{\partial t}\right|^{2}, c$ is independent of $\varepsilon$.

Proof Differentiate (1.15) with respect to $x_{s}, s=1,2, \ldots, N, N+1, x_{N+1}=t$, and sum up for $s$ after multiplying the resulting relation by $u_{\varepsilon x_{s}} \frac{S_{\eta}\left(\left|\operatorname{grad} u_{\varepsilon}\right|\right)}{\left|\operatorname{grad} u_{\varepsilon}\right|}$. In what follows, we simply denote $u_{\varepsilon}$ by $u$. Integrating over $\Omega$ yields

$$
\begin{gathered}
\int_{\Omega} \frac{\partial u_{x_{s}}}{\partial t} u_{x_{s}} \frac{S_{\eta}(|\operatorname{grad} u|)}{|\operatorname{grad} u|} d x=\int_{\Omega} \frac{\partial}{\partial t} \int_{0}^{|\operatorname{grad} u|} S_{\eta}(\tau) d \tau d x \\
=\frac{d}{d t} \int_{\Omega} I_{\eta}(|\operatorname{grad} u|) d x \\
\int_{\Omega} \frac{\partial}{\partial x_{s}}\left[\frac{\partial}{\partial x_{i}}\left(a^{i j}(u) \frac{\partial u}{\partial x_{j}}\right)\right] u_{x_{s}} \frac{S_{\eta}(|\operatorname{grad} u|)}{|\operatorname{grad} u|} d x \\
=\int_{\Omega} \frac{\partial}{\partial x_{i}}\left[a_{u}^{i j}(u) u_{x_{j}} u_{x_{s}}+a^{i j}(u) u_{x_{j} x_{s}}\right] u_{x_{s}} \frac{S_{\eta}(|\operatorname{grad} u|)}{|\operatorname{grad} u|} d x \\
=\int_{\Omega} \frac{\partial}{\partial x_{i}}\left(a_{u}^{i j}(u) u_{x_{j}} u_{x_{s}}\right) u_{x_{s}} \frac{S_{\eta}(|\operatorname{grad} u|)}{|\operatorname{grad} u|} d x \\
+\int_{\Omega} \frac{\partial}{\partial x_{i}}\left(a^{i j}(u) u_{x_{j} x_{s}}\right) u_{x_{s}} \frac{S_{\eta}(|\operatorname{grad} u|)}{|\operatorname{grad} u|} d x ;
\end{gathered}
$$

and, moreover,

$$
\begin{aligned}
& \int_{\Omega} \frac{\partial}{\partial x_{i}}\left(a_{u}^{i j}(u) u_{x_{j}} u_{x_{s}}\right) u_{x_{s}} \frac{S_{\eta}(|\operatorname{grad} u|)}{|\operatorname{grad} u|} d x \\
& =\sum_{s=1}^{N+1} \int_{\Omega} \frac{\partial}{\partial x_{i}}\left(a_{u}^{i j}(u) u_{x_{j}}\right) u_{x_{s}}^{2} \frac{S_{\eta}(|\operatorname{grad} u|)}{|\operatorname{grad} u|} d x+\int_{\Omega} a_{u}^{i j}(u) u_{x_{j}} \frac{\partial}{\partial x_{i}} I_{\eta}(|\operatorname{grad} u|) d x \\
& =\int_{\Omega} \frac{\partial}{\partial x_{i}}\left(a_{u}^{i j}(u) u_{x_{j}}\right)|\operatorname{grad} u| S_{\eta}(|\operatorname{grad} u|) d x-\int_{\Sigma} a_{u}^{i j}(u) u_{x_{i}} n_{j} I_{\eta}(|\operatorname{grad} u|) d \sigma
\end{aligned}
$$




$$
\begin{aligned}
& -\int_{\Omega} I_{\eta}(|\operatorname{grad} u|) \frac{\partial}{\partial x_{i}}\left(a_{u}^{i j}(u) u_{x_{j}}\right) d x \\
= & \int_{\Omega} \frac{\partial}{\partial x_{i}}\left(a_{u}^{i j}(u) u_{x_{j}}\right)\left[|\operatorname{grad} u| S_{\eta}(|\operatorname{grad} u|)-I_{\eta}(|\operatorname{grad} u|)\right] d x \\
& -\int_{\Sigma} a_{u}^{i j}(u) u_{x_{i}} n_{j} I_{\eta}(|\operatorname{grad} u|) d \sigma,
\end{aligned}
$$

where $\left\{n_{i}\right\}_{i=1}^{N}$ is the inner normal vector of $\Omega$ as before.

$$
\begin{aligned}
\int_{\Omega} & \frac{\partial}{\partial x_{i}}\left(a^{i j}(u) u_{x_{j} x_{s}}\right) u_{x_{s}} \frac{S_{\eta}(|\operatorname{grad} u|)}{|\operatorname{grad} u|} d x \\
= & \int_{\Omega} \frac{\partial}{\partial x_{i}}\left(a^{i j}(u) u_{x_{j} x_{s}}\right) \frac{\partial}{\partial \xi_{s}} I_{\eta}(|\operatorname{grad} u|) d x \\
= & -\int_{\Sigma} a^{i j}(u) u_{x_{i} x_{s}} n_{j} \frac{\partial}{\partial \xi_{s}} I_{\eta}(|\operatorname{grad} u|) d \sigma \\
& -\int_{\Omega} a^{i j}(u) \frac{\partial^{2} I_{\eta}(|\operatorname{grad} u|)}{\partial \xi_{s} \partial \xi_{p}} u_{x_{s} x_{i}} u_{x_{p} x_{j}} d x
\end{aligned}
$$

where $\xi_{s}=u_{x_{s}}$.

$$
\begin{aligned}
\varepsilon \int_{\Omega} \Delta u_{x_{s}} u_{x_{s}} \frac{S_{\eta}(|\operatorname{grad} u|)}{|\operatorname{grad} u|} d x= & -\varepsilon \int_{\Sigma} \frac{\partial I_{\eta}(|\operatorname{grad} u|)}{\partial x_{i}} n_{i} d \sigma \\
& -\varepsilon \int_{\Omega} \frac{\partial^{2} I_{\eta}(|\operatorname{grad} u|)}{\partial \xi_{s} \partial \xi_{p}} u_{x_{s} x_{i}} u_{x_{p} x_{i}} d x
\end{aligned}
$$

At the same time,

$$
\begin{aligned}
\int_{\Omega} & \nabla\left(\vec{b}^{\prime}(u) u_{x_{s}}\right) u_{x_{s}} \frac{S_{\eta}(|\operatorname{grad} u|)}{|\operatorname{grad} u|} d x \\
= & \sum_{i=1}^{N} \int_{\Omega} \frac{\partial}{\partial x_{i}}\left(b_{i}^{\prime}(u)\right)|\operatorname{grad} u| S_{\eta}(|\operatorname{grad} u|) d x+\sum_{i=1}^{N} \int_{\Omega} b_{i}^{\prime}(u) \frac{\partial I_{\eta}(|\operatorname{grad} u|)}{\partial x_{i}} d x \\
= & \sum_{i=1}^{N} \int_{\Omega} \frac{\partial}{\partial x_{i}}\left(b_{i}^{\prime}(u)\right)\left[|\operatorname{grad} u| S_{\eta}(|\operatorname{grad} u|)-I_{\eta}(|\operatorname{grad} u|)\right] d x \\
& -\int_{\Sigma} b_{i}^{\prime}(u) I_{\eta}(|\operatorname{grad} u|) n_{i} d \sigma .
\end{aligned}
$$

From (2.4)-(2.9), by the assumption $a^{i j}(0)=0$, we have

$$
\begin{aligned}
& \frac{d}{d t} \int_{\Omega} I_{\eta}(|\operatorname{grad} u|) d x \\
& =\int_{\Omega} \frac{\partial}{\partial x_{i}}\left(a_{u}^{i j}(u) u_{x_{j}}\right)\left[|\operatorname{grad} u| S_{\eta}(|\operatorname{grad} u|)-I_{\eta}(|\operatorname{grad} u|)\right] d x \\
& \quad-\int_{\Omega} a^{i j}(u) \frac{\partial^{2} I_{\eta}(|\operatorname{grad} u|)}{\partial \xi_{s} \partial \xi_{p}} u_{x_{s} x_{i}} u_{x_{p} x_{j}} d x-\varepsilon \int_{\Omega} \frac{\partial^{2} I_{\eta}(|\operatorname{grad} u|)}{\partial \xi_{s} \partial \xi_{p}} u_{x_{s} x_{i}} u_{x_{p} x_{i}} d x \\
& \quad+\sum_{i=1}^{N} \int_{\Omega} \frac{\partial}{\partial x_{i}}\left(b_{i}^{\prime}(u)\right)\left[|\operatorname{grad} u| S_{\eta}(|\operatorname{grad} u|)-I_{\eta}(|\operatorname{grad} u|)\right] d x
\end{aligned}
$$




$$
\begin{aligned}
& -\int_{\Sigma} a_{u}^{i j}(u) u_{x_{i}} n_{j} I_{\eta}(|\operatorname{grad} u|) d \sigma-\int_{\Sigma} b_{i}^{\prime}(u) I_{\eta}(|\operatorname{grad} u|) n_{i} d \sigma \\
& -\varepsilon \int_{\Sigma} \frac{\partial I_{\eta}(|\operatorname{grad} u|)}{\partial x_{i}} n_{i} d \sigma
\end{aligned}
$$

We shall use the fact that on $\Sigma$,

$$
b_{i}^{\prime}(u) \frac{\partial u}{\partial n} n_{i}=\varepsilon \Delta u+\frac{\partial}{\partial x_{i}}\left(a^{i j}(u) \frac{\partial u}{\partial x_{i}}\right), \quad u=0
$$

to calculate the surface integrals in (2.10). Formula (2.11) involves the derivatives on the boundary, let us give some explanation in the concept of local coordinates. Let $\delta_{0}>0$ be small enough such that

$$
E^{\delta_{0}}=\left\{x \in \bar{\Omega} ; \operatorname{dist}(x, \Sigma) \leq \delta_{0}\right\} \subset \bigcup_{\tau=1}^{n} V_{\tau},
$$

where $V_{\tau}$ is a region, on which one can introduce local coordinates

$$
y_{k}=F_{\tau}^{k}(x) \quad(k=1,2, \ldots, N),\left.\quad y_{N}\right|_{\Sigma}=0,
$$

with $F_{\tau}^{k}$ appropriately smooth and $F_{\tau}^{N}=F_{l}^{N}$, such that the $y_{N}$-axis coincides with the normal vector. Since the domain is bounded, there exists finite $V_{\tau}, \tau=1,2, \ldots, n$, such that $\bigcup_{\tau=1}^{n} V_{\tau} \supset \Sigma$.

Using these local coordinates on $V_{\tau}, \tau=1,2, \ldots, n$, by elementary computations (refer to [24]), we obtain on $\Sigma \cap V_{\tau}$

$$
u_{x_{i} x_{j}}=\sum_{k=1}^{N} u_{y_{N} y_{k}} F_{x_{i}}^{N} F_{x_{j}}^{k}+\sum_{k=1}^{N-1} u_{y_{N} y_{k}} F_{x_{i}}^{N} F_{x_{j}}^{k}+u_{y_{m}} F_{x_{i} x_{j}}^{m} .
$$

By this formula, what (2.11) means is clear.

Moreover, by (2.11), the surface integrals in (2.10) can be rewritten as

$$
\begin{aligned}
S= & -\left[\int_{\Sigma} b_{i}^{\prime}(u) I_{\eta}(|\operatorname{grad} u|) n_{i} d \sigma+\varepsilon \int_{\Sigma} \frac{\partial I_{\eta}(|\operatorname{grad} u|)}{\partial x_{i}} n_{i} d \sigma\right. \\
& \left.+\int_{\Sigma} a_{u}^{i j}(u) u_{x_{i}} n_{j} I_{\eta}(|\operatorname{grad} u|) d \sigma\right] \\
= & -\varepsilon \int_{\Sigma}\left[\frac{\partial I_{\eta}(|\operatorname{grad} u|)}{\partial x_{i}} n_{i}-\Delta u \frac{I_{\eta}(|\operatorname{grad} u|)}{\frac{\partial u}{\partial n}}\right] d \sigma \\
& +\int_{\Sigma} a^{i j}(u)\left[\frac{\partial I_{\eta}(|\operatorname{grad} u|)}{\partial x_{i}} n_{j}-u_{x_{i} x_{j}} \frac{I_{\eta}(|\operatorname{grad} u|)}{\frac{\partial u}{\partial n}}\right] d \sigma \\
= & -\varepsilon \int_{\Sigma}\left[\frac{\partial I_{\eta}(|\operatorname{grad} u|)}{\partial x_{i}} n_{i}-\Delta u \frac{I_{\eta}(|\operatorname{grad} u|)}{\frac{\partial u}{\partial n}}\right] d \sigma .
\end{aligned}
$$

Since

$$
\left.u_{x_{N+1}}\right|_{\Sigma}=\left.u_{t}\right|_{\Sigma}=0
$$


we have

$$
\lim _{\eta \rightarrow 0} S=\varepsilon \int_{\Sigma} \operatorname{sgn}\left(\frac{\partial u}{\partial \eta}\right)\left(u_{x_{s} x_{i}} n_{i} n_{S}-\Delta u\right) d \sigma .
$$

Noticing that

$$
u_{x_{i} x_{j}} n_{j} n_{i}=\frac{\sum_{k=1}^{N} u_{y_{N} y_{k}} F_{x_{i}}^{N} F_{x_{j}}^{k} F_{x_{j}}^{N} F_{x_{i}}^{N}}{\left|\operatorname{grad} F^{N}\right|^{2}}+\sum_{k=1}^{N-1} u_{y_{N} y_{k}} F_{x_{i}}^{k} F_{x_{j}}^{N}+\frac{u_{y_{m}} F_{x_{i} x_{j}}^{m} F_{x_{j}}^{N} F_{x_{i}}^{N}}{\left|\operatorname{grad} F^{N}\right|^{2}},
$$

in which $F^{k}=F_{\tau}^{k}$, by the fact that the normal vector is

$$
\vec{n}=\left(\frac{\partial F^{N}}{\partial x_{1}}, \ldots, \frac{\partial F^{N}}{\partial x_{N}}\right)=\operatorname{grad} F^{N},
$$

we have

$$
u_{x_{i} x_{j}} n_{j} n_{i}-\Delta u=u_{y_{m}}\left(\frac{F_{x_{i} x_{j}}^{m} F_{x_{j}}^{N} F_{x_{i}}^{N}}{\left|\operatorname{grad} F^{N}\right|^{2}}-F_{x_{i} x_{i}}^{m}\right) .
$$

Using Lemma 2.2, we are able to deduce that $\lim _{\eta \rightarrow 0} S$ can be estimated by $|\operatorname{grad} u|_{L_{1}(\Omega)}$.

Thus, letting $\eta \rightarrow 0$ in (2.10) and noticing that

$$
\lim _{\eta \rightarrow 0}\left[|\operatorname{grad} u| S_{\eta}(|\operatorname{grad} u|)-I_{\eta}(|\operatorname{grad} u|)\right]=0,
$$

using the fact that $\lim _{\eta \rightarrow 0} S$ can be estimated by $|\operatorname{grad} u|_{L_{1}(\Omega)}$, we have

$$
\frac{d}{d t} \int_{\Omega}|\operatorname{grad} u| d x \leq c_{1}+c_{2} \int_{\Omega}|\operatorname{grad} u| d x .
$$

By the well-known Gronwall lemma, we have

$$
\int_{\Omega}|\operatorname{grad} u| d x d t \leq c
$$

By (2.13), it is easy to show that

$$
\iint_{Q_{T}} a^{i j}(u) u_{x_{i}} u_{x_{j}} d x d t \leq c .
$$

Thus there exist a subsequence $\left\{u_{\varepsilon_{n}}\right\}$ of $u_{\varepsilon}$ and a function $u \in B V\left(Q_{T}\right) \cap L^{\infty}\left(Q_{T}\right)$ such that $u_{\varepsilon_{n}} \rightarrow u$ a.e. on $Q_{T}$; there exist functions $g^{i} \in L^{2}\left(Q_{T}\right)$ and a subsequence of $\{\varepsilon\}$ (we can simply denote this subsequence as $\{\varepsilon\}$ itself) such that when $\varepsilon \rightarrow 0$,

$$
\widehat{\gamma}^{i j} \frac{\partial u_{\varepsilon}}{\partial x_{j}} \rightarrow g^{i}, \quad \text { in } L^{2}\left(Q_{T}\right) .
$$

Proof of Theorem 1.2 We now prove that $u$ is a generalized solution of (1.1)-(1.5)-(1.19). Let $\varphi \in C^{2}\left(\overline{Q_{T}}\right), \varphi_{1} \geq 0, \operatorname{supp} \varphi \subset \bar{\Omega} \times(0, T),\left.\nabla \varphi_{1}\right|_{\Omega}=0$ and $\left\{n_{i}\right\}$ be the inner normal vector 
of $\Omega$. Multiply (1.15) by $\varphi_{1} S_{\eta}\left(u_{\varepsilon}-k\right)$ and integrate over $Q_{T}$ to obtain

$$
\begin{aligned}
& \iint_{Q_{T}} \frac{\partial u_{\varepsilon}}{\partial t} \varphi_{1} S_{\eta}\left(u_{\varepsilon}-k\right) d x d t \\
& \quad=\iint_{Q_{T}} \frac{\partial}{\partial x_{i}}\left(a^{i j}\left(u_{\varepsilon}\right) \frac{\partial u_{\varepsilon}}{\partial x_{j}}\right) \varphi_{1} S_{\eta}\left(u_{\varepsilon}-k\right) d x d t \\
& \quad+\varepsilon \iint_{Q_{T}} \Delta u_{\varepsilon} \varphi_{1} S_{\eta}\left(u_{\varepsilon}-k\right) d x d t+\sum_{i=1}^{N} \iint_{Q_{T}} \frac{\partial b_{i}\left(u_{\varepsilon}\right)}{\partial x_{i}} \varphi_{1} S_{\eta}\left(u_{\varepsilon}-k\right) d x d t .
\end{aligned}
$$

Let us calculate every term in (2.15) by the part integral method.

$$
\begin{aligned}
& \iint_{Q_{T}} \frac{\partial u_{\varepsilon}}{\partial t} \varphi_{1} S_{\eta}\left(u_{\varepsilon}-k\right) d x d t=-\iint_{Q_{T}} I_{\eta}\left(u_{\varepsilon}-k\right) \varphi_{1 t} d x d t, \\
& \varepsilon \iint_{Q_{T}} \Delta u_{\varepsilon} \varphi_{1} S_{\eta}\left(u_{\epsilon}-k\right) d x d t \\
& =-\varepsilon \int_{0}^{T} \int_{\Sigma} \nabla u_{\varepsilon} \cdot \vec{n} \varphi_{1} S_{\eta}\left(u_{\epsilon}-k\right) d t d \sigma \\
& -\varepsilon \iint_{Q_{T}} \nabla u_{\varepsilon}\left[S_{\eta}\left(u_{\varepsilon}-k\right) \nabla \varphi_{1}+\varphi_{1} S_{\eta}^{\prime}\left(u_{\varepsilon}-k\right) \nabla u_{\varepsilon}\right] d x d t \\
& =\varepsilon S_{\eta}(k) \int_{0}^{T} \int_{\Sigma} \nabla u_{\varepsilon} \cdot \vec{n} \varphi_{1} d t d \sigma-\varepsilon \iint_{Q_{T}} \nabla u_{\varepsilon} S_{\eta}\left(u_{\varepsilon}-k\right) \nabla \varphi_{1} d x d t \\
& -\varepsilon \iint_{Q_{T}}\left|\nabla u_{\varepsilon}\right|^{2} S_{\eta}^{\prime}\left(u_{\varepsilon}-k\right) \varphi_{1} d x d t \\
& \iint_{Q_{T}} \frac{\partial}{\partial x_{i}}\left(a^{i j}\left(u_{\varepsilon}\right) \frac{\partial u_{\varepsilon}}{\partial x_{j}}\right) \varphi_{1} S_{\eta}\left(u_{\varepsilon}-k\right) d x d t \\
& =S_{\eta}(k) \int_{0}^{T} \int_{\Sigma} a^{i j}\left(u_{\varepsilon}\right) \frac{\partial u_{\varepsilon}}{\partial x_{j}} n_{i} \varphi_{1} d t d \sigma \\
& -\iint_{Q_{T}} a^{i j}\left(u_{\varepsilon}\right) \frac{\partial u_{\varepsilon}}{\partial x_{j}}\left(S_{\eta}\left(u_{\varepsilon}-k\right) \varphi_{1 x_{i}}+\varphi_{1} S_{\eta}^{\prime}\left(u_{\varepsilon}-k\right) u_{\varepsilon x_{i}}\right) d x d t \\
& =S_{\eta}(k) \int_{0}^{T} \int_{\Sigma} a^{i j}\left(u_{\varepsilon}\right) \frac{\partial u_{\varepsilon}}{\partial x_{j}} n_{i} \varphi_{1} d t d \sigma-\iint_{Q_{T}} a^{i j}\left(u_{\varepsilon}\right) \frac{\partial u_{\varepsilon}}{\partial x_{j}} S_{\eta}\left(u_{\varepsilon}-k\right) \varphi_{1 x_{i}} d x d t \\
& -\iint_{Q_{T}} a^{i j}\left(u_{\varepsilon}\right) u_{\varepsilon x_{i}} u_{\varepsilon x_{j}} S_{\eta}^{\prime}\left(u_{\varepsilon}-k\right) \varphi_{1} d x d t
\end{aligned}
$$

and

$$
\begin{aligned}
& -\iint_{Q_{T}} a^{i j}\left(u_{\varepsilon}\right) \frac{\partial u_{\varepsilon}}{\partial x_{j}} S_{\eta}\left(u_{\varepsilon}-k\right) \varphi_{1 x_{i}} d x d t \\
& \quad=\iint_{Q_{T}} A_{\eta}^{i j}\left(u_{\varepsilon}, k\right) \varphi_{1 x_{i} x_{j}} d x d t+\int_{0}^{T} \int_{\Sigma} \nabla \varphi_{1 x_{i}} n_{j} A_{\eta}^{i j}\left(u_{\varepsilon}, k\right) d t d \sigma \\
& \iint_{Q_{T}} \frac{\partial b_{i}\left(u_{\varepsilon}\right)}{\partial x_{i}} \varphi_{1} S_{\eta}\left(u_{\varepsilon}-k\right) d x d t \\
& \quad=-\int_{0}^{T} \int_{\Sigma}\left[b_{i}\left(u_{\varepsilon}\right)-b(k)\right] n_{i} \varphi_{1} S_{\eta}\left(u_{\varepsilon}-k\right) d t d \sigma
\end{aligned}
$$




$$
\begin{aligned}
& -\iint_{Q_{T}}\left[b_{i}\left(u_{\varepsilon}\right)-b_{i}(k)\right]\left[\frac{\partial \varphi_{1}}{\partial x_{i}} S_{\eta}\left(u_{\varepsilon}-k\right)+\varphi_{1} S_{\eta}^{\prime}\left(u_{\varepsilon}-k\right) \frac{\partial u_{\varepsilon}}{\partial x_{i}}\right] d x d t \\
= & S_{\eta}(k) \int_{0}^{T} \int_{\Sigma} \varphi_{1}\left[b_{i}(0)-b_{i}(k)\right] n_{i} d \sigma d t-\iint_{Q_{T}} B_{\eta}^{i}\left(u_{\varepsilon}, k\right) \varphi_{1 x_{i}} d x d t .
\end{aligned}
$$

From (2.15)-(2.20), we have

$$
\begin{aligned}
& \iint_{Q_{T}} I_{\eta}\left(u_{\varepsilon}-k\right) \varphi_{1 t} d x d t+\iint_{Q_{T}} A_{\eta}^{i j}\left(u_{\varepsilon}, k\right) \varphi_{1 x_{i} x_{j}} d x d t-\iint_{Q_{T}} B_{\eta}^{i}\left(u_{\varepsilon}, k\right) \varphi_{1 x_{i}} d x d t \\
& \quad-\varepsilon \iint_{Q_{T}} \nabla u_{\varepsilon} \cdot \nabla \varphi_{1} S_{\eta}\left(u_{\varepsilon}-k\right) d x d t-\varepsilon \iint_{Q_{T}}\left|\nabla u_{\varepsilon}\right|^{2} S_{\eta}^{\prime}\left(u_{\varepsilon}-k\right) \varphi_{1} d x d t \\
& \quad-\iint_{Q_{T}} a^{i j}\left(u_{\varepsilon}\right) u_{\varepsilon x_{i}} u_{\varepsilon x_{i}} S_{\eta}^{\prime}\left(u_{\varepsilon}-k\right) \varphi_{1} d x d t+\varepsilon S_{\eta}(k) \int_{0}^{T} \int_{\Sigma} \nabla u_{\varepsilon} \cdot \vec{n} \varphi_{1} d t d \sigma \\
& +S_{\eta}(k) \int_{0}^{T} \int_{\Sigma} \frac{\partial}{\partial x_{i}}\left(a^{i j}\left(u_{\varepsilon}\right) \frac{\partial u_{\varepsilon}}{\partial x_{j}} n_{i} \varphi_{1} d t d \sigma+S_{\eta}(k) \int_{0}^{T} \int_{\Sigma} \varphi_{1 x_{i}} n_{j} A_{\eta}^{i j}(0, k) d t d \sigma\right. \\
& +S_{\eta}(k) \int_{0}^{T} \int_{\Sigma_{1 \eta k}}\left(b_{i}(0)-b_{i}(k)\right) n_{i} \varphi_{1} d t d \sigma \\
& +S_{\eta}(k) \int_{0}^{T} \int_{\Sigma_{2 \eta k}}\left(b_{i}(0)-b_{i}(k)\right) n_{i} \varphi_{1} d t d \sigma=0 .
\end{aligned}
$$

Taking $\varphi_{2} \in C^{2}\left(\overline{Q_{T}}\right),\left.\varphi_{1}\right|_{\partial \Omega \times[0, T]}=\left.\varphi_{2}\right|_{\partial \Omega \times[0, T]}, \operatorname{supp} \varphi_{2} \subset \bar{\Omega} \times(0, T)$,

$$
\begin{aligned}
& S_{\eta}(k) \int_{0}^{T} \int_{\Sigma} a^{i j}\left(u_{\varepsilon}\right) \frac{\partial u_{\varepsilon}}{\partial x_{j}} n_{i} \varphi_{1} d t d \sigma+\varepsilon S_{\eta}(k) \int_{0}^{T} \int_{\Sigma} \nabla u_{\varepsilon} \cdot \vec{n} \varphi_{1} d t d \sigma \\
&= S_{\eta}(k)\left\{-\varepsilon \iint_{Q_{T}} \frac{\partial u_{\varepsilon}}{\partial x_{i}} \frac{\partial \varphi_{2}}{\partial x_{i}} d x d t-\iint_{Q_{T}} a^{i j}\left(u_{\varepsilon}\right) \frac{\partial u_{\varepsilon}}{\partial x_{j}} \varphi_{2 x_{i}} d x d t\right. \\
&-\iint_{Q_{T}}\left[b_{i}\left(u_{\varepsilon}\right)-b_{i}(0)\right] \frac{\partial \varphi_{2}}{\partial x_{i}} d x d t \\
&\left.+\iint_{Q_{T}} u_{\varepsilon} \frac{\partial \varphi_{2}}{\partial t} d x d t-\int_{0}^{T} \int\left[b_{i}(0)-b_{i}(0)\right] n_{i} \varphi_{2} d t d \sigma\right\}, \\
& \iint_{Q_{T}} a^{i j}\left(u_{\varepsilon}\right) \frac{\partial u_{\varepsilon}}{\partial x_{j}} \varphi_{2 x_{i}} d x d t-\int_{0}^{T} \int_{\Sigma} a^{i j}(0) \frac{\partial \varphi_{2}}{\partial x_{i}} n_{j} d t d \sigma-\iint_{Q_{T}} a^{i j}\left(u_{\varepsilon}\right) \varphi_{2 x_{i} x_{j}} d x d t \\
&=-\iint_{Q_{T}} a^{i j}\left(u_{\varepsilon}\right) \varphi_{2 x_{i} x_{j}} d x d t .
\end{aligned}
$$

For $\left.\nabla \varphi_{1}\right|_{\Sigma}=0$ and $a^{i j}(0)=0$, from (2.21)-(2.23), we have

$$
\begin{aligned}
\iint_{Q_{T}} & I_{\eta}\left(u_{\varepsilon}-k\right) \varphi_{1 t} d x d t+\iint_{Q_{T}} A_{\eta}^{i j}\left(u_{\varepsilon}, k\right) \triangle \varphi_{1} d x d t-\iint_{Q_{T}} B_{\eta}^{i}\left(u_{\varepsilon}, k\right) \varphi_{1 x_{i}} d x d t \\
+ & S_{\eta}(k)\left[-\varepsilon \iint_{Q_{T}} \frac{\partial u_{\varepsilon}}{\partial x_{i}} \frac{\partial \varphi_{2}}{\partial x_{i}} d x d t+\iint_{Q_{T}} a^{i j}\left(u_{\varepsilon}\right) \varphi_{2 x_{i} x_{j}} d x d t\right. \\
& \left.-\iint_{Q_{T}} b_{i}\left(u_{\varepsilon}\right) \frac{\partial \varphi_{2}}{\partial x_{i}} d x d t+\iint_{Q_{T}} u_{\varepsilon} \frac{\partial \varphi_{2}}{\partial t} d x d t\right]
\end{aligned}
$$




$$
\begin{aligned}
& -\varepsilon \iint_{Q_{T}} \nabla u_{\varepsilon} \cdot \nabla \varphi_{1} S_{\eta}\left(u_{\varepsilon}-k\right) d x d t-\iint_{Q_{T}} a^{i j}\left(u_{\varepsilon}\right) u_{\varepsilon x_{i}} u_{\varepsilon x_{j}} S_{\eta}^{\prime}\left(u_{\varepsilon}-k\right) \varphi_{1} d x d t \\
& +S_{\eta}(k) \int_{0}^{T} \int_{\Sigma_{1 \eta k}}\left[\left(b_{i}(0)-b_{i}(k)\right] n_{i} \varphi_{1} d t d \sigma \geq 0 .\right.
\end{aligned}
$$

By Lemma 2.1,

$$
\begin{gathered}
\liminf _{\varepsilon \rightarrow 0} \iint_{Q_{T}} S_{\eta}^{\prime}\left(u_{\varepsilon}-k\right) a^{i j}\left(u_{\varepsilon}\right) \frac{\partial u_{\varepsilon}}{\partial x_{i}} \frac{\partial u_{\varepsilon}}{\partial x_{j}} \varphi_{1} d x d t \\
\geq \iint_{Q_{T}} \sum_{i=1}^{N}\left|g^{i}\right|^{2} S_{\eta}^{\prime}(u-k) \varphi_{1} d x d t .
\end{gathered}
$$

Let $\varepsilon \rightarrow 0$ in (2.24). By (2.25), we get (1.21). At the same time, (1.22) is naturally concealed in the limiting process.

The proof of (1.23) is similar to that in $[2,12]$, we omit the details here.

\section{Proof of Theorem 1.3}

Similar as the proof of Lemma 2 in [9], we can prove the following lemma.

Lemma 3.1 Let u be a solution of (1.1). Then

$$
\int_{u^{-}}^{u^{+}} \gamma^{i j}(s) d s \cdot v_{i}=0, \quad \text { a.e. }(x, t) \text { on } \Gamma^{u}, j=1,2, \ldots, N,
$$

is true in the sense of Hausdorff measure $H_{N}\left(\Gamma^{u}\right)$.

Proof of Theorem 1.3 Let $u, v$ be two entropy solutions of (1.1) with different initial values

$$
u(x, 0)=u_{0}(x), \quad v(x, 0)=v_{0}(x)
$$

and with the same homogeneous boundary value $\gamma u(x, t)=\gamma v(x, t)=0,(x, t) \in \Sigma_{1}$.

By Definition 1.1, for any $\varphi_{1}, \varphi_{2} \in C^{2}\left(\overline{Q_{T}}\right), \varphi_{1} \geq 0,\left.\varphi_{1}\right|_{\partial \Omega \times[0, T]}=\left.\varphi_{2}\right|_{\partial \Omega \times[0, T]}, \operatorname{supp} \varphi_{2}$, $\operatorname{supp} \varphi_{1} \subset \bar{\Omega} \times(0, T), \eta>0, k, l \in \mathbb{R}$, we have

$$
\begin{aligned}
& \iint_{Q_{T}}\left[I_{\eta}(u-k) \varphi_{1 t}-B_{\eta}^{i}(u, k) \varphi_{1 x_{i}}+A_{\eta}^{i j}(u, k) \varphi_{1 x_{i} x_{j}}-S_{\eta}^{\prime}(u-k) \sum_{i=1}^{N}\left|g^{i}(u)\right|^{2} \varphi_{1}\right] d x d t \\
& \quad+S_{\eta}(k)\left[b_{i}(0)-b_{i}(k)\right] \int_{0}^{T} \int_{\Sigma_{1 \eta k}} \varphi_{1} n_{i} d t d \sigma \\
& \quad+S_{\eta}(k) \iint_{Q_{T}}\left[u \varphi_{2 t}-\left(b_{i}(u)-b_{i}(0)\right) \varphi_{2 x_{i}}+A^{i j}(u) \varphi_{2 x_{i} x_{j}}\right] d x d t \geq 0, \\
& \iint_{Q_{T}}\left[I_{\eta}(v-l) \varphi_{1 \tau}-B_{\eta}^{i}(v, l) \varphi_{1 y_{i}}+A_{\eta}^{i j}(v, l) \varphi_{1 y_{i} y_{j}}-S_{\eta}^{\prime}(v-l) \sum_{i=1}^{N}\left|g^{i}(v)\right|^{2} \varphi_{1}\right] d y d \tau \\
& \quad+S_{\eta}(l)\left[\left(b_{i}(0)-b_{i}(l)\right] \int_{0}^{T} \int_{\Sigma_{1 \eta k}} \varphi_{1} n_{i} d \tau d \sigma\right. \\
& \quad+S_{\eta}(l) \iint_{Q_{T}}\left[v \varphi_{2 \tau}-\left(b_{i}(v)-b_{i}(0)\right) \varphi_{2 y_{i}}+A^{i j}(v) \varphi_{2 y_{i} y_{j}}\right] d y d \tau \geq 0 .
\end{aligned}
$$


Especially, if $\varphi_{1} \in C_{0}^{2}\left(Q_{T}\right), \varphi_{2} \equiv 0$, we have

$$
\begin{aligned}
& \iint_{Q_{T}}\left[I_{\eta}(u-k) \varphi_{1 t}-B_{\eta}^{i}(u, k) \varphi_{1 x_{i}}+A_{\eta}^{i j}(u, k) \varphi_{1 x_{i} x_{j}}\right. \\
& \left.-S_{\eta}^{\prime}(u-k) \sum_{i=1}^{N}\left|g^{i}(u)\right|^{2} \varphi_{1}\right] d x d t \geq 0, \\
& \iint_{Q_{T}}\left[I_{\eta}(v-l) \varphi_{1 \tau}-B_{\eta}^{i}(v, l) \varphi_{1 y_{i}}+A_{\eta}^{i j}(v, l) \varphi_{1 y_{i} y_{j}}\right. \\
& \left.\quad-S_{\eta}^{\prime}(v-l) \sum_{i=1}^{N}\left|g^{i}\right|^{2}(v) \varphi_{1}\right] d y d \tau \geq 0 .
\end{aligned}
$$

Let $\psi(x, t, y, \tau)=\phi(x, t) j_{h}(x-y, t-\tau)$. Here $\phi(x, t) \geq 0, \phi(x, t) \in C_{0}^{\infty}\left(Q_{T}\right)$, and

$$
\begin{aligned}
& j_{h}(x-y, t-\tau)=\omega_{h}(t-\tau) \prod_{i=1}^{N} \omega_{h}\left(x_{i}-y_{i}\right), \\
& \omega_{h}(s)=\frac{1}{h} \omega\left(\frac{s}{h}\right), \quad \omega(s) \in C_{0}^{\infty}(R), \quad \omega(s) \geq 0, \\
& \omega(s)=0 \quad \text { if }|s|>1, \int_{-\infty}^{\infty} \omega(s) d s=1 .
\end{aligned}
$$

Then we choose $k=v(y, \tau), l=u(x, t), \varphi_{1}=\psi(x, t, y, \tau)$ in (3.4), (3.5), integrate over $Q_{T}$ respectively, plus them together and get the following inequality

$$
\begin{aligned}
& \iint_{Q_{T}} \iint_{Q_{T}}\left[I_{\eta}(u-v)\left(\psi_{t}+\psi_{\tau}\right)-\left(B_{\eta}^{i}(u, v) \psi_{x_{i}}+B_{\eta}^{i}(v, u) \psi_{y_{i}}\right)\right. \\
& \left.\quad+A_{\eta}^{i j}(u, v) \psi_{x_{i} x_{j}}+A_{\eta}^{i j}(v, u) \psi_{y_{i} y_{j}}\right] \\
& \quad-S_{\eta}^{\prime}(u-v)\left(\sum_{i=1}^{N}\left|g^{i}(u)\right|^{2}+\sum_{i=1}^{N}\left|g^{i}(v)\right|^{2}\right) \psi d x d t d y d \tau \geq 0 .
\end{aligned}
$$

Clearly,

$$
\begin{aligned}
& \frac{\partial j_{h}}{\partial t}+\frac{\partial j_{h}}{\partial \tau}=0, \quad \frac{\partial j_{h}}{\partial x_{i}}+\frac{\partial j_{h}}{\partial y_{i}}=0, \quad i=1, \ldots, N ; \\
& \frac{\partial \psi}{\partial t}+\frac{\partial \psi}{\partial \tau}=\frac{\partial \phi}{\partial t} j_{h}, \quad \frac{\partial \psi}{\partial x_{i}}+\frac{\partial \psi}{\partial y_{i}}=\frac{\partial \phi}{\partial x_{i}} j_{h} .
\end{aligned}
$$

Noticing that

$$
\lim _{\eta \rightarrow 0} B_{\eta}^{i}(u, v)=\lim _{\eta \rightarrow 0} B_{\eta}^{i}(v, u)=\operatorname{sgn}(u-v)\left(b_{i}(u)-b_{i}(v)\right),
$$

as $\eta \rightarrow 0$, we have

$$
\begin{aligned}
& \iint_{Q_{T}} \iint_{Q_{T}}\left[B_{\eta}^{i}(u, v) \psi_{x_{i}}+B_{\eta}^{i}(v, u) \psi_{y_{i}}\right] d x d t d y d \tau \\
& \quad \rightarrow \iint_{Q_{T}} \iint_{Q_{T}} \operatorname{sgn}(u-v)\left[b_{i}(u)-b_{i}(v)\right] \phi_{x_{i}} j_{h} d x d t d y d \tau,
\end{aligned}
$$


as $h \rightarrow 0$, we have

$$
\begin{gathered}
\iint_{Q_{T}} \iint_{Q_{T}} \operatorname{sgn}(u-v)\left[b_{i}(u)-b_{i}(v)\right] \phi_{x_{i}} j_{h} d x d t d y d \tau \\
\rightarrow \iint_{Q_{T}} \operatorname{sgn}(u-v)\left[b_{i}(u)-b_{i}(v)\right] \phi_{x_{i}} d x d t
\end{gathered}
$$

For the third term and the fourth term on the left-hand side of (3.8), we have

$$
\begin{aligned}
& \iint_{Q_{T}}\left[A_{\eta}^{i j}(u, v) \psi_{x_{i} x_{j}}+A_{\eta}^{i j}(v, u) \psi_{y_{i} y_{j}}\right] d x d t d y d \tau \\
& =\iint_{Q_{T}} \iint_{Q_{T}}\left\{A_{\eta}^{i j}(u, v)\left(\phi_{x_{i} x_{j}} j_{h}+2 \phi_{x_{i}} j_{h x_{j}}+\phi j_{h x_{i} x_{j}}\right)+A_{\eta}^{i j}(v, u) \phi j_{h y_{i} y_{j}}\right\} d x d t d y d \tau \\
& =\iint_{Q_{T}} \iint_{Q_{T}}\left\{A_{\eta}^{i j}(u, v) \phi j_{h x_{i} x_{j}}+A_{\eta}^{i j}(u, v) \phi_{x_{i}} j_{h x_{j}}+A_{\eta}^{i j}(v, u) \phi_{x_{i}} j_{h y_{j}}\right\} d x d t d y d \tau \\
& \quad-\iint_{Q_{T}} \iint_{Q_{T}}\left\{a^{i j}(u) \widehat{S_{\eta}(u}-v\right) \frac{\partial u}{\partial x_{i}} \\
& \quad-\int_{u}^{v} a^{i j} \widehat{\left.s) S_{\eta}^{\prime}(s-v) d s \frac{\partial u}{\partial x_{i}} \phi j_{h x_{j}}\right\} d x d t d y d \tau,}
\end{aligned}
$$

where Definition 1.1 and formula (2.2) are used, i.e.,

$$
\begin{aligned}
& a^{i j}\left(\widehat{u) \widehat{S_{\eta}(u}}-v\right)=\int_{0}^{1} a^{i j}\left(s u^{+}+(1-s) u^{-}\right) S_{\eta}\left(s u^{+}+(1-s) u^{-}-v\right) d s, \\
& \left.\int_{u}^{v} a^{i j} \widehat{(s) \widehat{S_{\eta}^{\prime}(s}}-v\right) d s=\int_{0}^{1} \int_{s u^{+}+(1-s) u^{-}}^{v} a^{i j}(\sigma) S_{\eta}\left(\sigma-s u^{+}-(1-s) u^{-}\right) d \sigma d s .
\end{aligned}
$$

For the fifth term on the left-hand side of (3.8), we have

$$
\begin{aligned}
& \iint_{Q_{T}} \iint_{Q_{T}} S_{\eta}^{\prime}(u-v)\left(\sum_{i=1}^{N}\left|g^{i}(u)\right|^{2}+\sum_{i=1}^{N}\left|g^{i}(v)\right|^{2}\right) \psi d x d t d y d \tau \\
& =\sum_{i=1}^{N} \iint_{Q_{T}} \iint_{Q_{T}} S_{\eta}^{\prime}(u-v)\left(\left|g^{i}(u)\right|-\left|g^{i}(v)\right|\right)^{2} \psi d x d t d y d \tau \\
& \quad+2 \sum_{i=1}^{N} \iint_{Q_{T}} \iint_{Q_{T}} S_{\eta}^{\prime}(u-v) g^{i}(u) g^{i}(v) \psi d x d t d y d \tau \\
& =\sum_{i=1}^{N} \iint_{Q_{T}} \iint_{Q_{T}} S_{\eta}^{\prime}(u-v)\left(\left|g^{i}(u)\right|-\left|g^{i}(v)\right|\right)^{2} \psi d x d t d y d \tau \\
& \quad+2 \sum_{n=1}^{N} \iint_{Q_{T}} \iint_{Q_{T}} S_{\eta}^{\prime}(u-v) \partial_{x_{j}} \int_{v}^{u} \gamma^{n i}(s) d s \partial_{y_{j}} \int_{u}^{v} \gamma^{n j}(s) \psi d x d y d t d \tau .
\end{aligned}
$$

Now, by the properties of BV function,

$$
\begin{gathered}
\int_{Q_{T}} \int_{Q_{T}} \partial_{x_{i}} \partial_{y_{j}} \int_{v}^{u} r^{n i}(\delta) \int_{\delta}^{v} r^{n j}(\sigma) S_{\eta}^{\prime}(\sigma-\delta) d \sigma d \delta \psi d x d t d y d \tau \\
\quad=\int_{Q_{T}} \int_{Q_{T}} \psi \partial_{y_{j}} \int_{0}^{1} r^{i n}\left(s u^{+}+(1-s) u^{-}\right)
\end{gathered}
$$




$$
\begin{aligned}
& \times \int_{s u^{+}+(1-s) u^{-}}^{v} r^{n j}(\sigma) S_{\eta}^{\prime}\left(\sigma-s u^{+}-(1-s) u^{-}\right) d \sigma d s \frac{\partial u}{\partial x_{i}} d x d t d y d \tau \\
= & \int_{Q_{T}} \int_{Q_{T}} \psi \partial_{x_{i}} \int_{0}^{u} r^{i n}(\delta) d \delta \cdot \partial_{y_{j}} \int_{0}^{v} r^{n j}(\delta) d \delta S_{\eta}^{\prime}(v-u) d x d t d y d \tau \\
& \int_{Q_{T}} \int_{Q_{T}} \partial_{x_{i}} \partial_{y_{j}} \int_{v}^{u} r^{i n}(\delta) \int_{\delta}^{v} r^{n j}(\sigma) S_{\eta}^{\prime}(\sigma-\delta) d \sigma d \delta \psi d x d t d y d \tau \\
= & \int_{Q_{T}} \int_{Q_{T}} \psi \partial_{y_{j}} \int_{0}^{1} r^{i n}\left(s u^{+}+(1-s) u^{-}\right) \\
& \times \int_{s u^{+}+(1-s) u^{-}}^{v} r^{n j}(\sigma) S_{\eta}^{\prime}\left(\sigma-s u^{+}-(1-s) u^{-}\right) \frac{\partial u}{\partial x_{i}} d \sigma d s \\
= & \int_{Q_{T}} \int_{Q_{T}}^{v} \phi j_{h x_{j}} \int_{0}^{1} r^{i n}\left(s u^{+}+(1-s) u^{-}\right) \\
& \times \int_{s u^{+}+(1-s) u^{-}}^{v} r^{n j}(\sigma) S_{\eta}^{\prime}\left(\sigma-s u^{+}-(1-s) u^{-}\right) d \sigma d s \frac{\partial u}{\partial x_{i}} d x d t d y d \tau,
\end{aligned}
$$

we have

$$
\begin{aligned}
& -\int_{Q_{T}} \int_{Q_{T}}\left(a^{i j}\left(\widehat{u) \widehat{S_{\eta}(u}}-v\right) \frac{\partial u}{\partial x_{i}}+\int_{u}^{v} a^{i j}\left(\widehat{s) S_{\eta}^{\prime}(s}-u\right) d s \frac{\partial u}{\partial x_{i}}\right) j_{h x_{j}} \phi d x d t d y d \tau \\
& -2 \int_{Q_{T}} \int_{Q_{T}} S_{\eta}^{\prime}(u-v) \partial x_{i} \int_{0}^{u} r^{i n}(s) d s \cdot \partial y_{j} \int_{0}^{v} r^{n j}(s) d s \psi d x d t d y d \tau \\
& =-\int_{Q_{T}} \int_{Q_{T}}\left\{\int_{0}^{1} a^{i j}\left(s u^{+}+(1-s) u^{-}\right) S_{\eta}\left(s u^{+}+(1-s) u^{-}-v\right) d s\right. \\
& +\int_{0}^{1} \int_{s u^{+}+(1-s) u^{-}}^{v} a^{i j}(\sigma) S_{\eta}^{\prime}\left(\sigma-s u^{+}-(1-s) u^{-}\right) d \sigma d s \\
& -2 \int_{0}^{1} r^{i n}\left(s u^{+}+(1-s) u^{-}\right) \int_{s u^{+}+(1-s) u^{-}}^{v} r^{n j}(\sigma) \\
& \left.\times S_{\eta}^{\prime}\left(\sigma-s u^{+}-(1-s) u^{-}\right) d \sigma d s\right\} \frac{\partial u}{\partial x_{i}} j_{h x_{j}} \phi d x d t d y d \tau \\
& =\int_{Q_{T}} \int_{Q_{T}}\left\{\int_{0}^{1} \int_{s u^{+}+(1-s) u^{-}}^{v} r^{n j}\left(s u^{+}+(1-s) u^{-}\right)\right. \\
& \times r^{i n}\left(s u^{+}+(1-s) u^{-}\right) S_{\eta}^{\prime}\left(\sigma-s u^{+}-(1-s) u^{-}\right) d \sigma d s \\
& +\int_{0}^{1} \int_{s u^{+}+(1-s) u^{-}}^{v} r^{i n}(\sigma) r^{n j}(\sigma) S_{\eta}^{\prime}\left(\sigma-s u^{+}-(1-s) u^{-}\right) d \sigma d s \\
& -2 \int_{0}^{1} r^{i n}\left(s u^{+}+(1-s) u^{-}\right) \int_{s u^{+}+(1-s) u^{-}}^{v} r^{n j}(\sigma) \\
& \left.\times S_{\eta}^{\prime}\left(\sigma-s u^{+}-(1-s) u^{-}\right) d \sigma d s\right\} \frac{\partial u}{\partial x_{i}} j_{h x_{j}} \phi d x d t d y d \tau \\
& =\int_{Q_{T}} \int_{Q_{T}}\left\{\int _ { 0 } ^ { 1 } \int _ { s u ^ { + } + ( 1 - s ) u ^ { - } } ^ { v } \left\{r^{i n}\left(s u^{+}+(1-s) u^{-}\right)\left(r^{n j}\left(s u^{+}+(1-s) u^{-}\right)-r^{n j}(\sigma)\right)\right.\right. \\
& \left.-r^{n j}(\sigma)\left(r^{i n}\left(s u^{+}+(1-s) u^{-}\right)-r^{i n}(\sigma)\right)\right\} \\
& \left.\times S_{\eta}^{\prime}\left(\sigma-s u^{+}-(1-s) u^{-}\right) d \sigma d s\right\} \frac{\partial u}{\partial x_{i}} j_{h x_{j}} \phi d x d t d y d \tau \text {. }
\end{aligned}
$$


Since

$$
\begin{gathered}
\int_{s u^{+}+(1-s) u^{-}}^{v}\left\{r^{i n}\left(s u^{+}+(1-s) u^{-}\right)\left(r^{n j}\left(s u^{+}+(1-s) u^{-}\right)-r^{n j}(\sigma)\right)\right. \\
\left.-r^{n j}(\sigma)\left(r^{i n}\left(s u^{+}+(1-s) u^{-}\right)-r^{i n}(\sigma)\right)\right\} S_{\eta}^{\prime}\left(\sigma-s u^{+}-(1-s) u^{-}\right) d \sigma
\end{gathered}
$$

is uniformly bounded and leads to 0 as $\eta \rightarrow 0$, we have, when $\eta \rightarrow 0$,

$$
\begin{aligned}
& -\int_{Q_{T}} \int_{Q_{T}}\left(a^{i j}\left(\widehat{u) S_{\eta}(u}-v\right) \frac{\partial u}{\partial x_{i}}-\int_{u}^{v} a^{i j}\left(\widehat{s)} \widehat{S_{\eta}^{\prime}(s}-u\right) d s \frac{\partial u}{\partial x_{i}}\right) j_{h x_{j}} \phi d x d t d y d \tau \\
& -2 \int_{Q_{T}} \int_{Q_{T}} S_{\eta}^{\prime}(u-v) \partial x_{i} \int_{0}^{u} r^{i n}(s) d s \cdot \partial y_{j} \int_{0}^{v} r^{n j}(s) d s \psi d x d t d y d \tau \rightarrow 0 .
\end{aligned}
$$

At the same time, noticing that $\lim _{\eta \rightarrow 0} A_{\eta}^{i j}(u, v)=\lim _{\eta \rightarrow 0} A_{\eta}^{i j}(v, u)=\operatorname{sgn}(u-v)\left(A^{i j}(u)-\right.$ $\left.A^{i j}(v)\right)$, we have

$$
\lim _{\eta \rightarrow 0}\left(A_{\eta}^{i j}(u, v) \phi_{x_{i}} j_{h x_{i}}+A_{\eta}^{i j}(v, u) \phi_{x_{i}} j_{h y_{i}}\right)=0 .
$$

Combining (3.8)-(3.13), and letting $\eta \rightarrow 0, h \rightarrow 0$, we get

$$
\begin{gathered}
\int_{Q_{T}}\left\{|u(x, t)-v(x, t)| \phi_{t}+\operatorname{sgn}(u-v)\left(A^{i j}(u)-A^{i j}(v)\right) \phi_{x_{i} x_{j}}\right. \\
\left.-\operatorname{sgn}(u-v)\left(b_{i}(u)-b_{i}(v)\right) \phi_{x_{i}}\right\} d x d t \geq 0 .
\end{gathered}
$$

Let $\delta_{\varepsilon}$ be the mollifier as usual. If $y=\left(x_{1}, \ldots, x_{N}\right)$, then

$$
\delta(y)= \begin{cases}\frac{1}{A} e^{\frac{1}{|y|^{2}-1}} & \text { if }|y|<1 \\ 0 & \text { if }|y| \geq 1\end{cases}
$$

where

$$
A=\int_{B_{1}(0)} e^{\frac{1}{|y|^{2}-1}} d x .
$$

For any given $\varepsilon>0, \delta_{\varepsilon}(y)$ is defined as

$$
\delta_{\varepsilon}(y)=\frac{1}{\varepsilon^{N}} \delta\left(\frac{y}{\varepsilon}\right)
$$

Especially, we can choose $\phi$ in (3.14) by

$$
\phi(x, t)=\omega_{\lambda \varepsilon}(x) \eta(t)
$$

where $\eta(t) \in C_{0}^{\infty}(0, T), \omega_{\lambda \varepsilon}(x)$ is defined as follows. Let $\omega_{\lambda}(x) \in C_{0}^{2}(\Omega)$ be defined as follows: for any given small enough $0<\lambda, 0 \leq \omega_{\lambda} \leq 1,\left.\omega\right|_{\partial \Omega}=0$ and

$$
\omega_{\lambda}(x)=1, \quad \text { if } d(x)=\operatorname{dist}(x, \partial \Omega) \geq \lambda
$$


When $0 \leq d(x) \leq \lambda$,

$$
\omega_{\lambda}(d(x))=1-\frac{(d(x)-\lambda)^{2}}{\lambda^{2}} .
$$

Then

$$
\begin{aligned}
& \omega_{\lambda \varepsilon}=\omega_{\lambda} * \delta_{\varepsilon}(d), \\
& \omega_{\lambda \varepsilon}^{\prime}(d)=\int_{\{|s|<\varepsilon\} \cap\{0<d-s<\lambda\}} \omega_{\lambda}^{\prime}(d-s) \delta_{\varepsilon}(s) d s \\
& =-\int_{\{|s|<\varepsilon\} \cap\{0<d-s<\lambda\}} \frac{2(d-s-\lambda)}{\lambda^{2}} \delta_{\varepsilon}(s) d s, \\
& \left|\omega_{\lambda \varepsilon}^{\prime}(d)\right| \leq \frac{c}{\lambda}, \\
& \omega_{\lambda \varepsilon}^{\prime \prime}(d)=\omega_{\lambda}^{\prime \prime} * \delta_{\varepsilon}(d)=-\frac{2}{\lambda^{2}} \int_{\{|s|<\varepsilon\} \cap\{0<d-s<\lambda\}} \delta_{\varepsilon}(s) d s .
\end{aligned}
$$

Now,

$$
\begin{aligned}
\phi_{x_{i} x_{j}} & =\eta(t)\left(\omega_{\lambda \varepsilon}(d(x))\right)_{x_{i} x_{j}} \\
& =\eta(t)\left(\omega_{\lambda \varepsilon}^{\prime}(d) d_{x_{i}}\right)_{x_{j}} \\
& =\eta(t)\left[\omega_{\lambda \varepsilon}^{\prime \prime}(d) d_{x_{i}} d_{x_{j}}+\omega_{\lambda \varepsilon}^{\prime}(d) d_{x_{i} x_{j}}\right] \\
& =\eta(t)\left[-\frac{2}{\lambda^{2}} d_{x_{i}} d_{x_{j}} \int_{\{|s|<\varepsilon\} \cap\{0<d-s<\lambda\}} \delta_{\varepsilon}(s) d s+\omega_{\lambda \varepsilon}^{\prime}(d) d_{x_{i} x_{j}}\right]
\end{aligned}
$$

using the conditions $\left|d_{x_{i} x_{j}}\right| \leq c$, and using the fact that $|\nabla d|=1$, noticing that

$$
\operatorname{sgn}(u-v)\left(A^{i j}(u)-A^{i j}(v)\right) d_{x_{i}} d_{x_{j}}=|u-v| a^{i j}(\zeta) d_{x_{i}} d_{x_{j}} \geq 0,
$$

where $\zeta \in(v, u)$. Then by (1.26), from (3.14), we have

$$
\int_{Q_{T}}|u(x, t)-v(x, t)| \phi_{t} d x d t+c \int_{0}^{T} \int_{\Omega_{\langle} \Omega_{\lambda}} \eta(t)\left|\omega_{\lambda \varepsilon}^{\prime}(d)\right||u-v| d x d t \geq 0,
$$

where $\Omega_{\lambda}=\{x \in \Omega: d(x, \partial \Omega)<\lambda\}$.

According to the definition of trace (3.3), let $\lambda \rightarrow 0$ in (3.17). By (3.15)-(3.16), we have

$$
\underset{\Sigma_{2} \times(0, T)}{\operatorname{ess} \sup }|f(x, t)-g(x, t)|+\int_{Q_{T}}|u(x, t)-v(x, t)| \eta_{t}^{\prime} d x d t \geq 0 .
$$

Let $0<s<\tau<T$, and

$$
\eta(t)=\int_{\tau-t}^{s-t} \alpha_{\varepsilon}(\sigma) d \sigma, \quad \varepsilon<\min \{\tau, T-s\} .
$$

Here $\alpha_{\varepsilon}(t)$ is the kernel of the mollifier with $\alpha_{\varepsilon}(t)=0$ for $t \notin(-\varepsilon, \varepsilon)$. Then

$$
c \underset{\Sigma_{2} \times(0, T)}{\operatorname{essup}}|f(x, t)-g(x, t)|+\int_{0}^{T}\left[\alpha_{\varepsilon}(t-s)-\alpha_{\varepsilon}(t-\tau)\right]|u-v|_{L^{1}(\Omega)} d t \geq 0 .
$$


Let $\varepsilon \rightarrow 0$. Then

$$
|u(x, \tau)-v(x, \tau)|_{L^{1}(\Omega)} \leq|u(x, s)-v(x, s)|_{L^{1}(\Omega)}+\underset{\Sigma_{2} \times(0, T)}{\operatorname{ess} \sup }|f(x, t)-g(x, t)|
$$

and the desired result follows by letting $s \rightarrow 0$.

4 The case of $\Sigma_{1}=\emptyset$

If $\Sigma_{1}=\emptyset$, the solution of equation (1.1) is completely controlled by the initial value condition. Now, we should give the following definition.

Definition 4.1 A function $u$ is said to be the entropy solution of equation (1.1) with initial value (1.5) if

1. $u$ satisfies

$$
u \in B V\left(Q_{T}\right) \cap L^{\infty}\left(Q_{T}\right), \quad \frac{\partial}{\partial x_{i}} \int_{0}^{u} \sqrt{a(s)} d s \in L^{2}\left(Q_{T}\right) .
$$

2. For any $\varphi \in C_{0}^{2}\left(Q_{T}\right), \varphi \geq 0$, for any $k \in \mathbb{R}$, for any small $\eta>0$, $u$ satisfies

$$
\begin{aligned}
& \iint_{Q_{T}}\left[I_{\eta}(u-k) \varphi_{t}-B_{\eta}^{i}(u, k) \varphi_{x_{i}}\right. \\
& \left.+A_{\eta}^{i j}(u, k) \frac{\partial \varphi}{\partial x_{i}} \frac{\partial \varphi}{\partial x_{j}}-S_{\eta}^{\prime}(u-k) \sum_{j=1}^{N}\left|g^{j}\right|^{2} \varphi\right] d x d t \geq 0 .
\end{aligned}
$$

3. The initial value is true in the sense of

$$
\lim _{t \rightarrow 0} \int_{\Omega}\left|u(x, t)-u_{0}(x)\right| d x=0, \quad \text { a.e. } x \in \Omega .
$$

Similarly as in the proofs of Theorems 1.2 and 1.3, we can prove the following theorems, and we omit the details here.

Theorem 4.2 Suppose that $A^{i j}(s)$ is $C^{3}, b_{i}(s)$ is $C^{2}, u_{0}(x) \in L^{\infty}(\Omega)$, and suppose that

$$
a^{i j}(0)=0 \text {. }
$$

Then equation (1.1) with initial value condition (1.5) has an entropy solution in the sense of Definition 4.1.

Theorem 4.3 Suppose that $A^{i j}(s), b_{i}(s)$ is $C^{1}$. Let $u, v$ be solutions of equation (1.1) with different initial values $u_{0}(x), v_{0}(x) \in L^{\infty}(\Omega)$, respectively. Suppose that the distance function $d(x)=\operatorname{dist}(x, \Sigma)$ satisfies (2.17), and that

$$
\gamma u(x, t)=f(x, t), \quad \gamma v=g(x, t), \quad(x, t) \in \Sigma \times(0, T) .
$$

Then

$$
\int_{\Omega}|u(x, t)-v(x, t)| d x \leq \int_{\Omega}\left|u_{0}-v_{0}\right| d x+\underset{(x, t) \in \Sigma \times(0, T)}{\operatorname{ess} \sup }|f(x, t)-g(x, t)| .
$$




\section{Competing interests}

The author declares that they have no competing interests.

\section{Acknowledgements}

The paper is supported by NSF of China (No. 11371297), supported by SF of Xiamen University of Technology, China.

Received: 29 October 2014 Accepted: 6 January 2015 Published online: 31 January 2015

\section{References}

1. Oleinik, OA, Samokhin, VN: Mathematical Models in Boundary Layer Theorem. Chapman \& Hall/CRC, London (1999)

2. Vol'pert, Al, Hudjaev, SI: On the problem for quasilinear degenerate parabolic equations of second order. Mat. Sb. 3, 374-396 (1967) (in Russian)

3. Zhao, J: Uniqueness of solutions of quasilinear degenerate parabolic equations. Northeast. Math. J. 1(2), 153-165 (1985)

4. Brezis, $H$, Crandall, MG: Uniqueness of solutions of the initial value problem for $u_{t}-\Delta \varphi(u)=0$. J. Math. Pures Appl. 58, 153-163 (1979)

5. Kružkov, SN: First order quasilinear equations in several independent variables. Math. USSR Sb. 10, $217-243$ (1970)

6. Cockburn, B, Gripenberg, G: Continuous dependence on the nonlinearities of solutions of degenerate parabolic equations. J. Differ. Equ. 151, 231-251 (1999)

7. Vol'pert, Al: BV space and quasilinear equations. Mat. Sb., 73, 255-302 (1967)

8. Zhuoqun, W, Junning, Z, Jinxue, Y, Huilai, L: Nonlinear Diffusion Equations. World Scientific, Singapore (2001)

9. Zhao, J, Zhan, H: Uniqueness and stability of solution for Cauchy problem of degenerate quasilinear parabolic equations. Sci. China Ser. A 48, 583-593 (2005)

10. Chen, GQ, Perthame, B: Well-posedness for non-isotropic degenerate parabolic-hyperbolic equations. Ann. Inst. Henri Poincaré, Anal. Non Linéaire 20(4), 645-668 (2003)

11. Chen, GQ, DiBenedetto, E: Stability of entropy solutions to the Cauchy problem for a class of nonlinear hyperbolic-parabolic equations. SIAM J. Math. Anal. 33(4), 751-762 (2001)

12. Karlsen, $\mathrm{KH}$, Risebro, $\mathrm{NH}$ : On the uniqueness of entropy solutions of nonlinear degenerate parabolic equations with rough coefficient. Discrete Contin. Dyn. Syst. 9(5), 1081-1104 (2003)

13. Bendahamane, $\mathrm{M}$, Karlsen, $\mathrm{KH}$ : Reharmonized entropy solutions for quasilinear anisotropic degenerate parabolic equations. SIAM J. Math. Anal. 36(2), 405-422 (2004)

14. Carrillo, J: Entropy solutions for nonlinear degenerate problems. Arch. Ration. Mech. Anal. 147, $269-361$ (1999)

15. Li, Y, Wang, Q: Homogeneous Dirichlet problems for quasilinear anisotropic degenerate parabolic-hyperbolic equations. J. Differ. Equ. 252, 4719-4741 (2012)

16. Lions, PL, Perthame, B, Tadmor, E: A kinetic formation of multidimensional conservation laws and related equations. J. Am. Math. Soc. 7, 169-191 (1994)

17. Kobayasi, $\mathrm{K}$, Ohwa, $\mathrm{H}$ : Uniqueness and existence for anisotropic degenerate parabolic equations with boundary conditions on a bounded rectangle. J. Differ. Equ. 252, 137-167 (2012)

18. Tricomi, F: Sulle equazioni lineari alle derivate parziali di secondo ordine, di tipo misto. Rend. Reale Accad. Lincei Ser. 5 $14,134-247$ (1923)

19. Keldyš, MV: On certain cases of degeneration of elliptic type on the boundary of a domain. Dokl. Akad. Nauk SSSR 77, 181-183 (1951)

20. Fichera, G: Sulle equazioni differenziali lineari ellittico-paraboliche del secondo ordine. Atti Accad. Naz. Lincei, Rend. Cl. Sci. Fis. Mat. Nat. (8) 5, 1-30 (1956)

21. Fichera, G: On a unified theory of boundary value problems for elliptic-parabolic equations of second order. In: Boundary Problems, Differential Equations, pp. 97-120. University of Wisconsin Press, Madison (1960)

22. Oleĭnik, OA: A problem of Fichera. Dokl. Akad. Nauk SSSR 154, 1297-1300 (1964); English transl.: Sov. Math. Dokl. 5 , 1129-1133 (1964)

23. Oleĭnik, OA: Linear equations of second order with nonnegative characteristic form. Mat. Sb. (N.S.) 69, 111-140 (1966): English transl.: Transl. Am. Math. Soc. 65(2), 167-199 (1967)

24. Wu, Z, Zhao, J: The first boundary value problem for quasilinear degenerate parabolic equations of second order in several variables. Chin. Ann. Math., Ser. B 4(3), 319-358 (1983)

25. Evans, LC: Weak Convergence Methods for Nonlinear Partial Differential Equations. CBMS Regional Conference Series in Mathematics, vol. 74 (1998)

26. Gu, L: Second Order Parabolic Partial Differential Equations. Xiamen University Press, Xiamen (2004) (in Chinese) 